Journal of Computer Science 4 (12): 1003-1011, 2008

ISSN 1549-3636

(C) 2008 Science Publications

\title{
Design and Analysis of Virtual Bus Transport Using Synchronous Digital Hierarchy/Synchronous Optical Networking
}

\author{
${ }^{1}$ Rosilah Hassan, ${ }^{2}$ James Irvine and ${ }^{2}$ Ian Glover \\ ${ }^{1}$ Department of Computer Science, Faculty of Information Science and Technology, \\ University Kebangsaan Malaysia, 43600 UKM Bangi Selangor, Malaysia \\ ${ }^{2}$ Mobile and RF Communications Group, Institute for Communications and Signal Processing, \\ University of Strathclyde, Royal College Building, 204 George Square, \\ G1 1XW, Glasgow, Scotland, United Kingdom
}

\begin{abstract}
This study presented a new data link layer design for the virtual bus architecture. The virtual bus is a hierarchical access network offered a broadcast capability for data and signaling. The proposed of the study is to support Quality of Service (QoS) in network. During study, the most promising technology capable of delivering full service access at high data rates is Synchronous Digital Hierarchy (SDH)/Synchronous Optical Networking (SONET). For that reason, we develop $\mathrm{SDH} / \mathrm{SONET}$ as a physical layer for the virtual bus network. The main goal of this investigation was to study the performance of transmission medium and the overall performance of the network. We exemplify a Multiple Access Protocol (MAP) with Generic Frame Procedure (GFP) for transmitting IP network protocol datagram over SDH/SONET. This is a novel method for transmitting MAP with GFP. The protocol adds multi-terminal access capability to an inherently point-to-point link. It enabled the design of seamless networking environments using SDH/SONET as the transmission medium for Metropolitan Area Network (MAN). The new frame mapping protocol is known as Virtual Bus Transport (VBT). The performance of multiple traffic generators in a virtual bus network is of major interest for mobile network provider. We used OMNeT simulation tool to evaluate the result. Through the simulation it proved that the VBT scheduling technique offers better treatment mainly to the higher Qos requirement traffic.
\end{abstract}

Key words: Qos, SDH/SONET, Multiple access protocol, generic frame procedure, virtual bus transport

\section{INTRODUCTION}

In next generation systems, it is likely that data traffic will dominate and the trend is towards Internet Protocol (IP) based networks will continue. The trend towards IP based data services means that the Data Link Layer (DLL) used must be able to support IP and transport the resulting packets efficiently. The virtual bus ${ }^{[1,2]}$ is a new interconnection methodology that simplifies signaling in a mobile Radio Access Network (RAN). In this investigation, we design a new data link layer for the virtual bus architecture that uses Synchronous Digital Hierarchy (SDH) or Synchronous Optical Networking (SONET) for physical transport. The SDH/SONET standard has been chosen as it has a number of properties, which are particularly attractive for a radio access network. It supports data rates from
51.84 Mbps to $13.22 \mathrm{Gbps}$, with 155 Mbps (STM-1 or OC-3) and 622 Mbps (STM-4 or OC-12) being the most common. Quality of Service (QoS) capabilities and the facility to aggregate various data rates make it the preferred choice for Metropolitan Access Networks (MAN). Among other system architecture issues, the selection of a suitable Medium Access Control (MAC) protocol plays a key role. The protocol must be designed such that it can support existing applications and evolving services and provide the quality of services guarantees offered by other access technologies.

\section{System and network architecture:}

Virtual bus architecture: The virtual bus $\operatorname{architecture}^{[3,4]}$ is a hierarchical access network structure that has been proposed to simplify network

Corresponding Author: Rosilah Hassan, Department of Computer Science, Faculty of Information Science and Technology, University Kebangsaan Malaysia, 43600 UKM Bangi Selangor, Malaysia

Tel: +60389216181 Fax: +603 89257954 
signaling. A Cell Site Switch (CSS) controls the connection to each Virtual Bus (VB) segment and a VB Backbone interconnects all the CSS. A Local Exchange (LE) unit provides the interface to the Core IP network, Fig. 1. A distributed hierarchical database is used to support advanced routing of both signaling and traffic. Interconnecting the base stations by VB segments allows signaling to be efficiently transferred, where appropriate, using only a single segment. The virtual bus also provides a broadcast capability for data and signaling, simplifying control and handover.

Motivation for virtual bus transport: Among other system architecture issues, the selection of a suitable MAC protocol plays a key role. The protocol must be designed such that it can support existing applications and evolving services and provide the quality of service guarantees offered by other access technologies. The aim is to propose a new data link layer, which is simple, distributed, hierarchical and robust. Our motivation to design a new data link layer is the need to fulfill the constraint of the virtual bus architecture.

One of the main characteristics of the virtual bus architecture is a broadcast capability, which makes this architecture different from other radio access networks. Packet over SDH/SONET has been implemented before as described in $^{[5]}$. However, Point-to-Point (PPP) was used to establish a direct connection between two nodes. Here we design a data link layer that has a capability to send packets from one node to multiple nodes at one time. The increased data traffic requires technologies to transport IP packets to the physical layer.

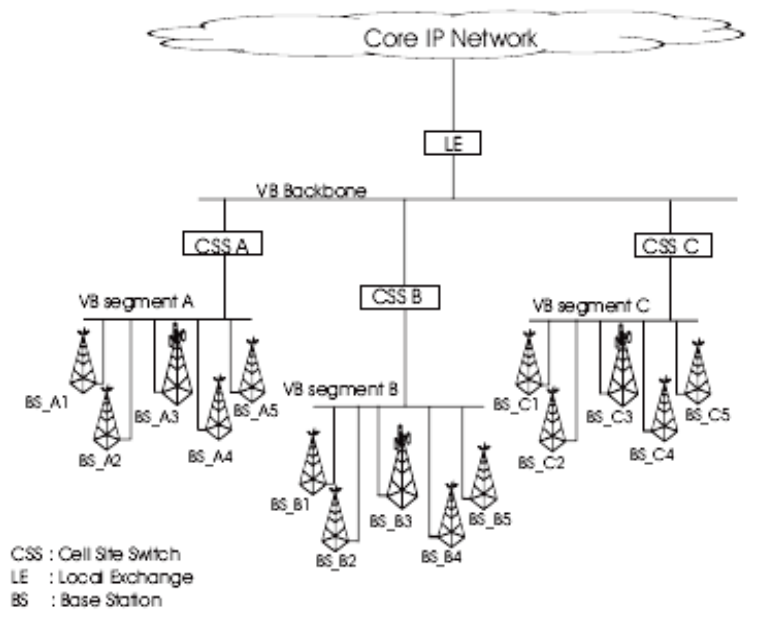

Fig. 1: Virtual Bus Architecture
We illustrate the concept of multiple access protocol over SDH/SONET for transmitting IP network protocol datagram with broadcast capability in the virtual bus network. This mapping is of IP over virtual bus transport over SDH/SONET. The traditional mechanisms for carrying IP over SDH/SONET are shown in Fig. 2, along with the proposal for VBT. VBT uses GFP for mapping VB packet into SDH/SONET. Two conditions will apply:

- GFP provides frame delineation

- GFP adds idle frames depending on the rate of the VB packet and the rate of the SDH/SONET signal

Access network: There are a variety of protocols for transmitting IP across networks. The evolution of SDH/SONET mapping started with $\mathrm{ATM}^{[5]}$ and continued with Packet over SONET (PoS), Data over SONET (DoS), Ethernet over SONET (EoS) and Resilient Packet Ring (RPR). All of these technologies use SDH/SONET for physical layer transmission and the standard SDH/SONET optical carrier interfaces can support the usual data rates. The PoS interface ${ }^{[6]}$, consists of IP/PPP/HDLC over SDH/SONET where the IP datagrams are encapsulated in point-to-point protocol packets. The encapsulated datagrams are then framed using high-level data link control and finally mapped into the SDH/SONET synchronous payload envelope. Currently, PoS is popular in backbone links between core IP routers running at 2.5 and $10 \mathrm{Gbps}^{[7]}$ whilst IP over ATM is still acceptable in lower speed access networks where bandwidth management is essential. Soon, however, next generation framing protocols such as GFP will transmit IP over GFP over SDH/SONET and directly over fiber and will become widely deployed in future high-speed optical networks.

Link layer protocol: The data link layer defines how data is formatted for transmission and how access to the network is controlled. This layer has been divided by the IEEE 802 standards committee into two sub layers: Media Access Control (MAC) and Logical Link Control (LLC). When we consider the link layer as provider, the user layer is the network layer,

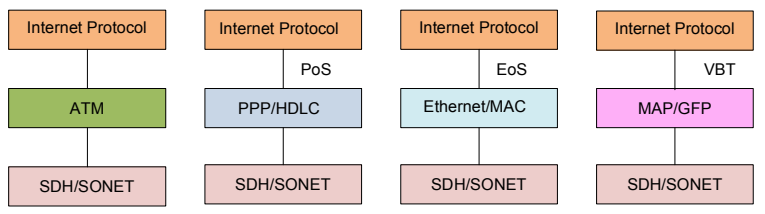

Fig. 2: Traditional IP over SDH/SONET transport mechanism and the proposed idea 
which wants to send its data packets from one network address to another. It expects the link layer to accept these addresses and the data and whatever additional information is needed, such as packet lifetime, type of service required and to package this all up in such a way that the link layer can give it to an appropriate hardware layer as its agent. Examples of data link protocols are Ethernet for local area networks and Point-to-Point Protocol (PPP), High-Level Data Link Control (HDLC), Advanced Data Communication Control Procedures (ADCCP) for point-to-point connections and MAP.

Multiple access protocol: Multiple access protocol ${ }^{[8,9]}$ is a high-speed link layer protocol that provides a multiple access capability. The core of the medium access control in such an environment is the multiple access protocol since many subscribers share the same cable or fiber for transmissions ${ }^{[10]}$. In this work, we propose a multiple access protocol using centralized and distributed channel access control techniques to provide QoS guarantees for multimedia services. A component called a frame switch in a generic framing procedure allows multiple nodes (host and routers) to be connected together in a star topology to form a LAN. MAP uses time division multiple access. A multiple access protocol that dynamically allocates the bandwidth to a large number of nodes must be efficient and flexible. It may employ static assignment, random access, demand assignment or some combination of these. Here we are concerned with the issues of a distributed multiple access protocol with a generic framing procedure.

Generic framing procedure: Generic Framing Procedure (GFP) ${ }^{[11-13]}$ is a new standard that has been developed to overcome data transport inefficiencies and deficiencies inherent in the existing ATM and PoS protocols. HDLC protocols like PoS need nearly twice the genuine data bandwidth to transmit the packet. GFP reduces the overhead by using information in its core header for frame delimitation. The GFP consist of the Core Header and the Payload Header as shown in Fig. 3. The core header comprises a 2-byte field that specifies the length of the GFP frame's payload area in bytes and Cyclic Redundancy Check (CRC-16) code over this length field. The PLI and the cHEC are used as a frame delineation mechanism. Both may vary frame-to-frame. The flexible payload size from 4 to 65,535 bytes is a significant advantage of GFP. The frame format is described as:

\section{GFP core header:}

- Payload Length Indicator (PLI): 2 bytes, allowing for a frame length of up to 65,535 byte
- Core HEC (cHEC): CRC-16 protects against errors in count field (single-bit error correction + multibit error detection)

\section{GFP payload area:}

- Payload header: (4-64) byte. (4 byte mandatory, 60 byte optional extension)

- Payload type

- Payload FCS Indicator (PFI)

- Payload Type Identifier (PTI)

- $\quad$ Extension Header Identifier (EXI)

- User Payload Identifier (UPI)

- Payload information field: Contains the frame PDU

- Payload Frame Check Sequence (FCS): Optional 4-octet long frame check sequence. It contains a CRC-32 check sequence that protects the contents of the GFP payload information field

To cater for all mapping requirements, two mapping modes are formally defined in GFP:

- $\quad$ Framed mapped (GFP-F): GFP-F supports variable-sized packet lengths of framed data, where a single client data frame (e.g., IP packet, Ethernet MAC) is mapped directly into a single GPF-F frame. This requires a MAC protocol to terminate layer 2. It can be used for Ethernet, PPP and HDLC-like protocols where efficiency and flexibility are important. To perform the encapsulation process it is necessary to receive the complete client packet. This requirement increases latency, however, making GFP-F inappropriate for time-sensitive protocols

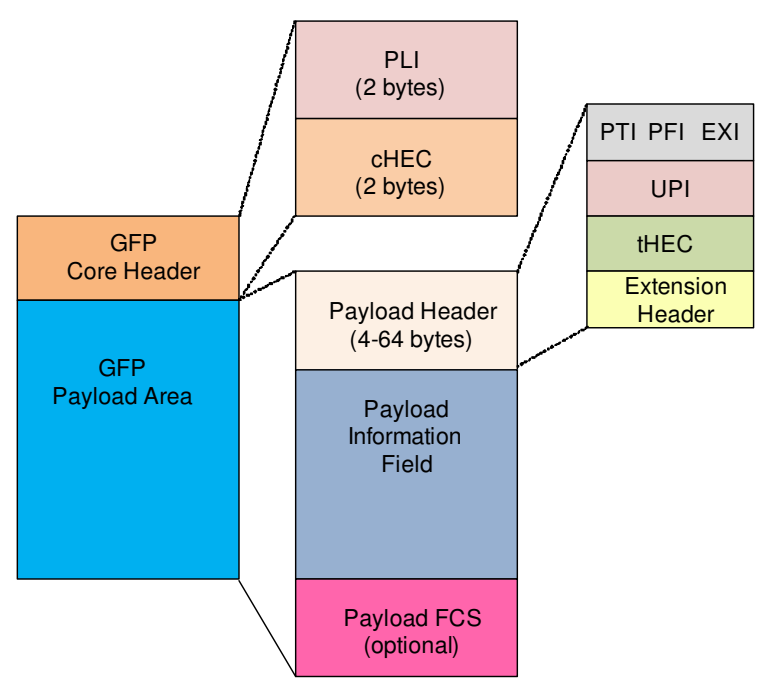

Fig. 3: Generic framing procedure frame format 
- Transparent mapped (GFP-T): GFP-T supports fixed packet lengths. GFP-T does not require MAC functionality, as it is notionally transparent to the protocol transmitted. The frame is transmitted immediately without waiting for the entire client data packet to be received. GFP-F is the suitable mode for mapping IP over SDH/SONET. This mode maps a single, entire, client frame into one GFP frame. In the particular context of the problem addressed here a single IP frame is mapped into a single GFP frame

SDH/SONET: SDH/SONET is a high bandwidth carrier service, which can provide a transport facility for any network technology. SONET is the ANSI standard used in the United States for synchronous data transmission on optical media. The international equivalent of SONET used in Europe is the ITU standard Synchronous Digital Hierarchy (SDH). Several alternatives can be considered for implementation in the virtual bus network. The virtual bus concept is independent of the fundamental physical and data link technologies. A transmission medium model is necessary, however, to investigate virtual bus performance. Much previous work has been reported on the implementation of a virtual bus using the IEEE 802.6 Standard known as Distributed Queue Dual Bus Metropolitan Area Network (DQDB MAN $)^{[3,14]}$. A number of technologies such as Gigabit Ethernet and $\mathrm{SDH} / \mathrm{SONET}$, offer support at high data rates. As these high-speed technologies become available to end users, high-speed applications will develop quickly in the network environment. $\mathrm{In}^{[2]}$, we have settled on a $\mathrm{SDH} / \mathrm{SONET}$ as a physical layer medium. One of the reasons for choosing SDH/SONET solutions is because this standard is the most successful and widely spread technology. Generally high-speed digital backbones are based on SDH/SONET technology SDH/SONET has a layered architecture and mainly deals with the physical layer of the OSI model.

\section{MATERIALS AND METHODS}

Design of the virtual bus transport: We now propose a new layer two for the virtual bus network suitable for metropolitan area networks. Virtual bus transport is thus a data link layer protocol for transmitting IP packets over SDH/SONET. Two-stage encapsulation is defined. Firstly, IP packets are encapsulated by a multi access protocol. Secondly, they are encapsulated to form a frame similar to the generic framing protocol. Packets are thus taken from the data link layer via virtual concatenation and turned into SDH/SONET frames. Multiple end nodes are connected to a GFP frame switch as shown in Fig. 4. The frame switch forwards packets between nodes and provides the multiple access capability. Each port on a switch has a unique identifier within the switch. A node connected to a switch port inherits the address of the port. The node address is therefore the same as the port identifier and is unique within the switch.

Virtual Bus Transport Stack: The virtual bus protocol stack for operating IP over SDH/SONET is shown in Fig. 5. Originally, the VB architecture consists of four interacting domains: Local Exchange (LE), Cell Site Switch (CSS), Base Station (BS) and Mobile Host (MH). The CSS and LE act as a gateway used for packet switched domains depending on where handover occurs. Since the focus of this study is VBT, however, we make no assumptions about the air interface in order to emphasize the data link design.

Figure 6 shows the virtual bus protocol stack and the signaling protocol structure between the nodes in the network. All packets arriving at the gateway are sent via the base station to the end user.

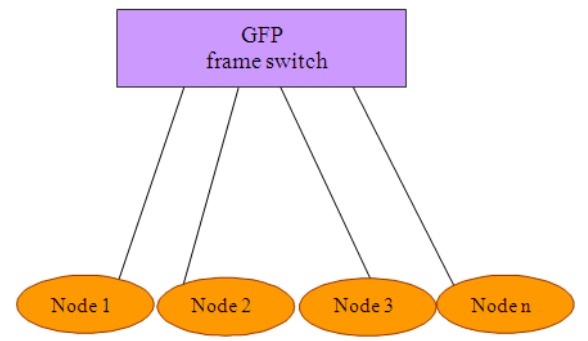

Fig. 4: Point-to-Multipoint Configuration

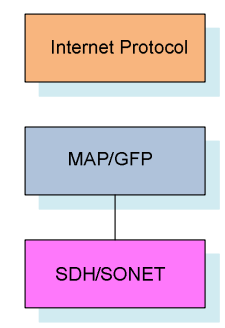

Fig. 5: Virtual Bus Protocol Stack for IP over SDH/SONET

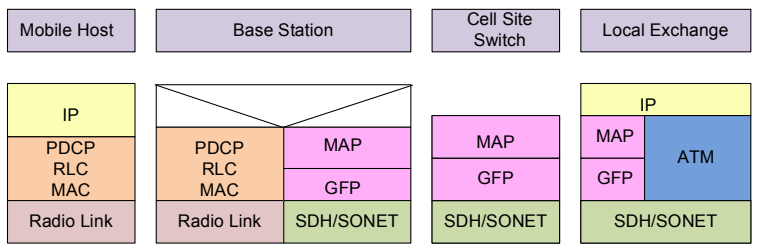

Fig. 6: Virtual Bus protocol model for IP packet data 


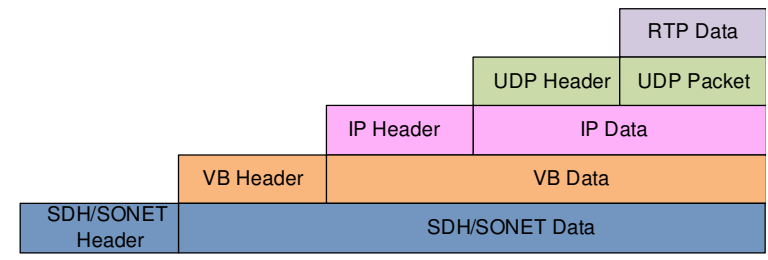

Fig. 7: Sample encapsulation of data in the Virtual Bus Network

In the GFP downlink the data stream is split between multiple cell site switches and the resulting sub-streams divided between multiple base stations as previously shown in Fig. 1. The principal function of the LE is to provide switching for user traffic. It hosts the databases and network management functions and also handles routing and mobility management.

Virtual bus data encapsulation: Since voice traffic is time-sensitive the connection-oriented Transmission Control Protocol (TCP) is not used for VoIP packets and the connectionless User Datagram Protocol (UDP) is employed instead. Since UDP cannot control the order in which packets are received, the actual voice content is encapsulated via the Real-time Transport Protocol (RTP). The voice transmission architecture is thus RTP over UDP over IP. The proposed packet generator therefore provides the overall data unit structure shown in Fig. 7. According to the VoIP paradigm and in order to avoid introducing large conversion delays, voice samples are compressed and packetized into segments whose length does not usually exceed 20 bytes. This payload is time-stamped using RTP, which introduces a 12-bytes header. The resulting segment is then carried by a UDP datagram, which adds a further 8-byte header. While RTP provides the facilities for time synchronization, UDP allows several streams to be multiplexed together into a connectionless logical channel. Encapsulation in an IP datagram incurs yet a further 20 byte header. The overall result is a large (40 byte) overhead.

Payload mapping over IP: In order to utilize different sized containers efficiently payload mapping, i.e., a systematic method for inserting and removing the payload from a SDH/SONET container, is required. The relationship between an IP frame and GFP is shown in Fig. 8. Many mappings between a higher layer Protocol Data Unit (PDU) and a GFP PDU are possible. Here, the mapping is considered end on IP over SDH/SONET.

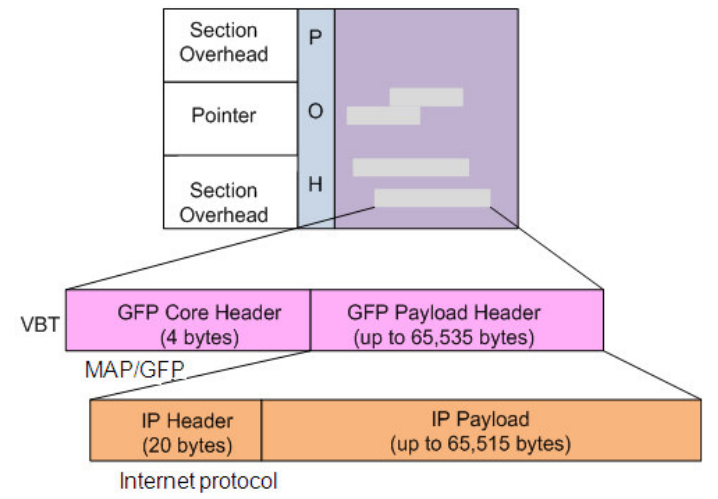

Fig. 8: IP over VBT over SDH/SONET

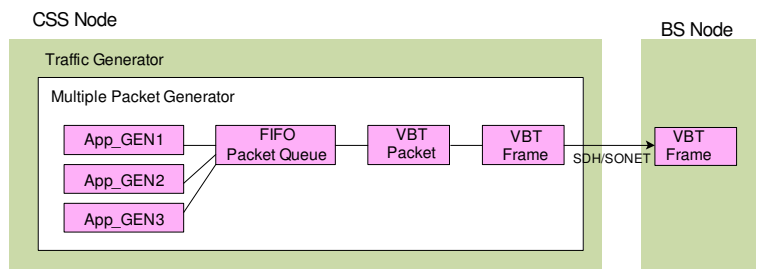

Fig. 9: Simulation model for multiple packet generators

An IP packet payload can be up to 655,150 bytes. In this study, however, we assume only 40 bytes since our interest is in the effectiveness of VoIP when this fixed size of IP packet is applied. The maximum payload for VBT is 65,535 bytes while the core header is 4 bytes. This is the required payload size when using the GFP frame in the link layer. The basic STS-1 SDH/SONET frame structure consists of 810 bytes, transmitted 8,000 times $\mathrm{sec}^{-1}$ to form a $51.840 \mathrm{Mbps}$ data rate ${ }^{[14]}$. For that reason, one SDH/SONET frame is sent every 125 micro sec. Figure 8, IP over VBT over SDH/SONET uses a time division multiplexed frame structure as GFP.

Performance assessment of the virtual bus transport:

Verification for VBT: In order to validate and verify the virtual bus transport, we have modeled it using $\mathrm{OMNeT}++^{[15]}$, an object oriented modular discrete event simulator. Initial verification will consider the output when a single VoIP call is simulated using multiple packet generators. The fixed packet size of VoIP is used as a parameter. End-to-end throughput, packet loss and packet delay performance will be determined and compared with other transport strategies such as IP over ATM and resilient packet ring.

Simulation scenario: In this scenario we use a multiple packet generator. The multiple packet generators are used to evaluate the VBT performance at the CSS node and BS node. Figure 9 shows the 
simulation model. Throughput and packet delay are evaluated in the BS node.

Throughout the simulation, the traffic generators are always 'ON' (there is no waiting state). The IP packet generator will transmit over VBT where VBT throughput and VBT delay will be identified. We used $\mathrm{SDH} / \mathrm{SONET}$ as the transport medium. In this scenario, multiple IP traffic: voice traffic, data traffic, video traffic and multimedia messaging service are included. For voice, a fixed IP packet size is 40 bytes long including headers with Constant Bit Rate (CBR). The IP link capacity is $8 \mathrm{Mbps}$ and all the IP sources were set as UDP. For that reason, the inter-arrival time for packet generators is different for voice and data traffic. In this scenario we used a real-time traffic application only. We exploit two kinds of traffic model: constant bit rate and variable bit rate. Data traffic is assumed to be constant bit rate whereas voice traffic and video traffic is variable bit rate.

Simulation parameter: The total channel capacity for the traffic generator is set to $8 \mathrm{Mbps}$ and the total simulation time in this experiment is $300 \mathrm{sec}$ as indicated by the steady-state analysis in the previous section. The IP packet size for each traffic type is different. In this research, we will make an assumption that the traffic mix is $60 \%$ Conversational sessions, $30 \%$ Streaming and 5\% Interactive and Background. The idea behind this is to have highest voice traffic in the network, followed by MMS traffic and almost equivalent traffic for data and video. From the bandwidth utilization in the traffic generator, ten traffic flows of application packet generators are used, as shown in Table 1 and 2. If $\mu$ is the packet $\sec ^{-1}, \mathrm{x}$ is the data rates in bps and $\mathrm{m}$ is IP packet size in bits:

$$
\mu=\frac{x}{m}
$$

Therefore, the inter-arrival time $(\lambda)$ in seconds is as:

$$
\lambda=\frac{1}{\mu}
$$

The guideline of the packet inter-arrival time is shown in Table 1 . However, this was the maximum value as not supposed to exceed the total channel capacity used in the simulation.

In this scenario the traffic generator still the same as at the CSS node. The CSS then broadcasts the VBT frame to all the BS nodes on the virtual bus segment.
Table 1: Traffic specification of the flows used in the scenario

\begin{tabular}{llcl}
\hline Packet generator & Traffic flow & $\begin{array}{l}\text { Packet size } \\
\text { (bytes) }\end{array}$ & $\begin{array}{l}\text { Inter-arrival } \\
\text { time (sec) }\end{array}$ \\
\hline App-Gen1 & Voice 1 & 40 & 0.003 \\
App-Gen2 & Voice 2 & 40 & 0.003 \\
App-Gen3 & Voice 3 & 40 & 0.003 \\
App-Gen4 & Voice 4 & 40 & 0.003 \\
App-Gen5 & Voice 5 & 40 & 0.003 \\
App-Gen6 & Data 1 & 1024 & 0.300 \\
App-Gen7 & Data 2 & 1024 & 0.300 \\
App-Gen8 & Video 1 & 1362 & 0.500 \\
App-Gen9 & Video 2 & 1362 & 0.500 \\
App-Gen10 & MMS 1 & 30000 & 1.000 \\
\hline
\end{tabular}

\begin{tabular}{lll} 
Table 2: Parameter used & & \\
\hline Parameter & Traffic types & Notes \\
\hline Traffic types & Voice traffic -40 & bytes \\
& Data traffic -1024 & \\
& Video traffic -1362 & \\
& MMS traffic - 30000 & Mbps \\
Channel capacity & 8 & second \\
Time-to-stop-gen & 300 & second \\
Simulation time & 301 &
\end{tabular}

For simplicity of simulation when multiple packets have been generated, we add a parameter to the application generator called time to stop gen in order to make sure no packets are left in the queue when the simulation time is reached. Hence, Table 2 shows the summed parameter used in the simulation for scenario 2. Since voice traffic has the largest number of packets in the network, the graph for this type of traffic is separated from other traffic.

\section{RESULTS}

Figure 10 shows the output of the voice traffic generator. It demonstrates that the voice traffic is increasing as the network load of the system increases. Both Fig. 10 and 11 show the packet behavior from the different generators. Figure 10 is the throughput for five-application generators for voice traffic whereas Fig. 10 is throughput from the total of network load for two-generators of data traffic, two-generators for video and a single generator for MMS traffic. It can be seen that in terms of number of packets, voice traffic dominates other types of traffic. This is because voice packets are much smaller than the other traffic types.

Figure 12 shows traffic load in terms of bits/sec for each traffic types whereas Fig. 13 shows the average packet or frame against network load of VBT packet and VBT frame. The VBT packet and frame sizes are very different, up to 65535 bytes and 810 bytes respectively. The number of VBT packets is constant with time and does not rely on network loads, but the contents of VBT packet is related to the network load. 


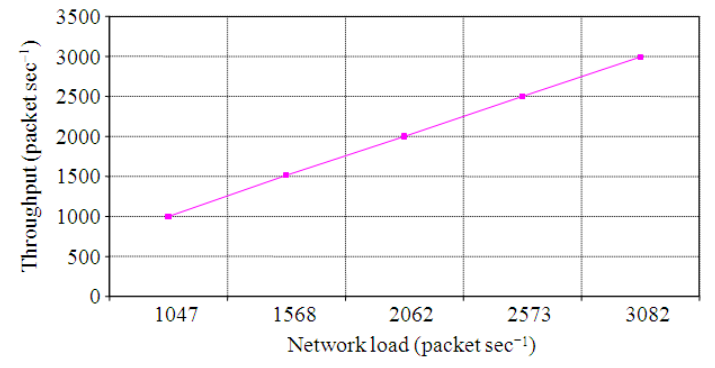

Fig. 10: Multiple packet generators for five voice traffic

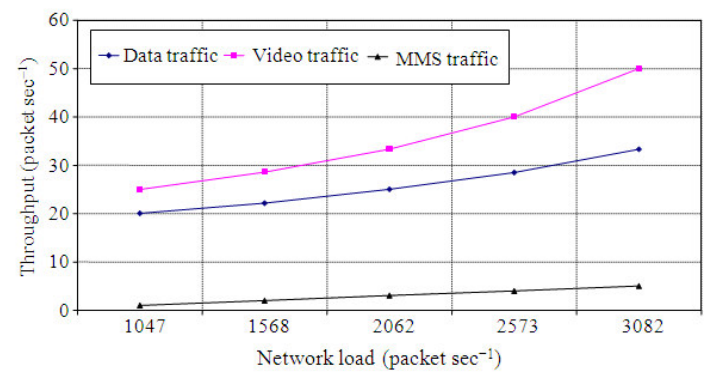

Fig. 11: Multiple packet generators for two data, two video and one MMS traffic

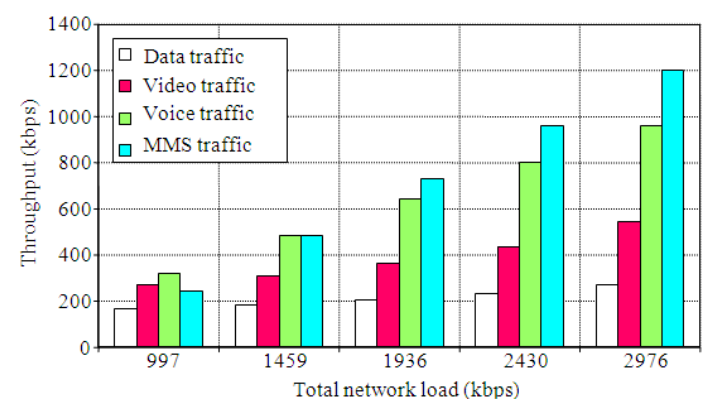

Fig. 12: Load Delivered by the Network

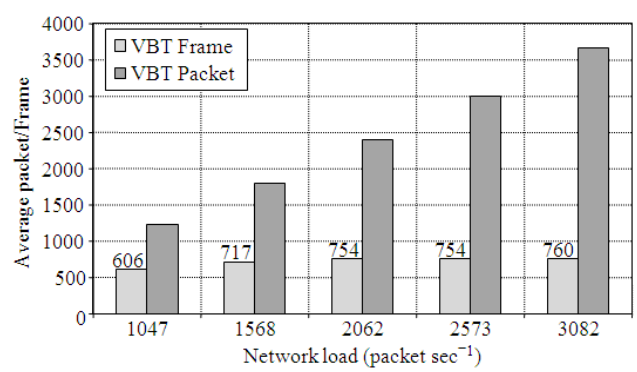

Fig. 13: VBT Packet and VBT Frame

Figure 14 shows the average of VBT packet delay and Fig. 15 shows the average of VBT frame delay. This delay is taken in the traffic generator with 4 types of queues.

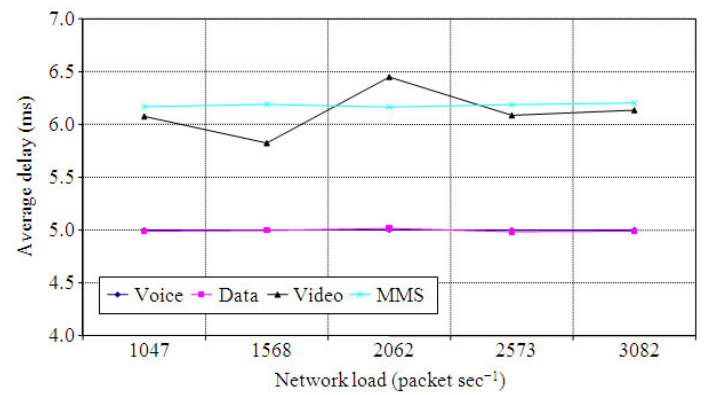

Fig. 14: VBT packet delay

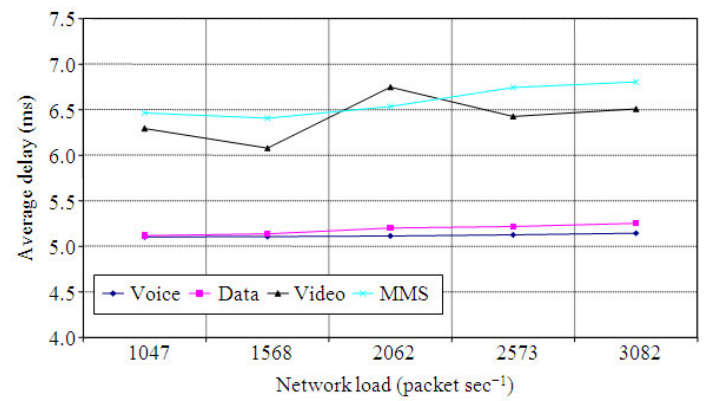

Fig. 15: VBT frame delay

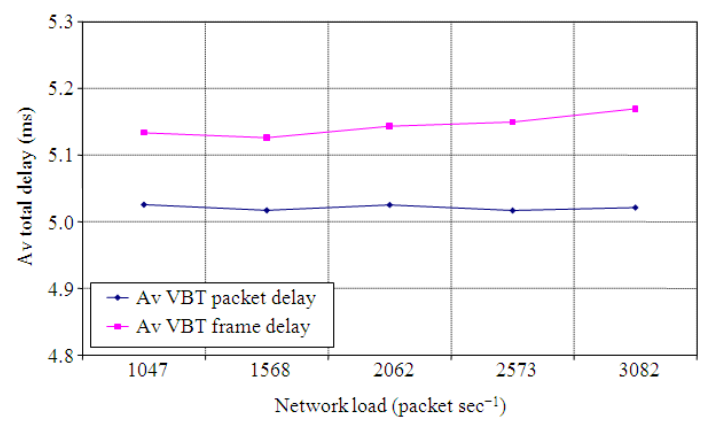

Fig. 16: Delay in the network

Figure 16 shows the two types of delays in the network. It shows that the VBT frame delay is increased as the network load is increased. However, the VBT packet delay remains steady for all cases. As the conclusion of this scenario, we observe the throughputs of flows in VBT. From four types of traffic in the simulation, voice has the highest volume, which is 60 percent of the total traffic that has been generated in the network. This is followed by MMS traffic that is 30 percent whereas both data traffic and video traffic are maintained at 4 percent of the total packets that we have generated. Figure 17 shows the delay at the base station as an end node for this scenario. Voice application typically requires an end-to-end delay not greater than $150 \mathrm{~ms}$ for acceptable quality ${ }^{[16]}$. 


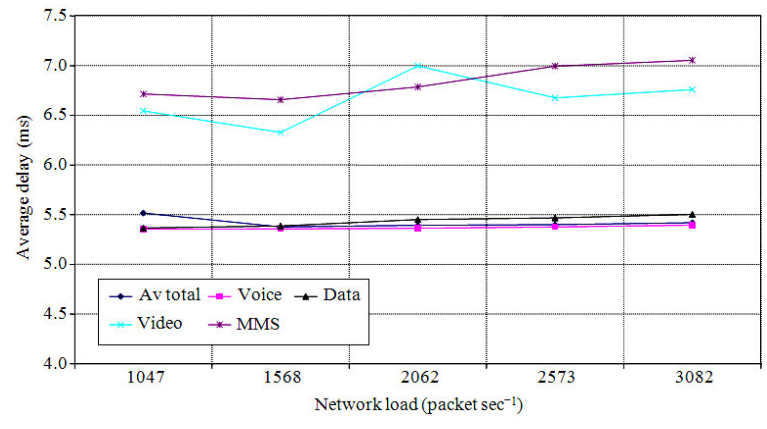

Fig. 17: Delay at the base station

\section{DISCUSSION}

A new data link layer which we call virtual bus transport or VBT has been presented. It is specifically designed for the virtual bus network architecture. As a transport protocol, virtual bus transport operates at layer one and layer two of the seven layer OSI networking model, delivering its data packets to any device connected to the network. Packets are transmitted using IP over VBT over SDH/SONET. VBT uses a multiple access protocol and generic framing procedure to transfer a packet to the physical layer of the network. By their nature, real-time multimedia applications such as VoIP and video conferencing are sensitivities to latency. In order to function properly, therefore some level of guaranteed quality of service must be maintained. The outcome of this simulation will make our work easier for the next scenario evaluation. We will use the same packet generator behavior for quality of service evaluation as our future study.

\section{CONCLUSION}

To conclude, this study makes a contribution in three areas. Firstly by replacing the existing transmission medium, DQDB MAN physical layer for the virtual bus with SDH/SONET. Secondly, by designing of new transmission medium of the network known as virtual bus transport. Lastly we investigate the performance on QoS support in the virtual bus using simulation for multiple packet generators.

\section{ACKNOWLEDGMENT}

The researchers acknowledge the financial support received from the Ministry of Higher Education, Malaysia via University Kebangsaan Malaysia (UKM).

\section{REFERENCES}

1. Hassan, R., J. Irvine and I. Glover, 2007. Designing of data link layer for virtual bus architecture. Proceeding of the 9th International Conference on Advanced Communication Technology, Feb. 12-14, IEEE Xplore Press, Gongwan-Do Republic of Korea, pp: 1978-1982. DOI: 10.1109/ICACT.2007.358759

2. Hassan, R. and J. Irvine, 2006. Implementation of the virtual bus architecture. Proceeding of the Conference on Malaysian Research Group (MRG) International, June 19-21, Manchester, pp: 464-470, http://www.justbooks.co.uk/dir/i/Proceedings_of_t he_Malaysian_Research_Group_International_Con ference_2006-19th/1905732031/

3. Bodic, G., Le. D. Girma, J. Irvine and J. Dunlop, 2000. Virtual bus architecture for hierarchical cellular system. Proceeding of PIMRC, Sept. 1822, London, pp: 861-865. DOI: 10.1109/PIMRC.2000.881545

4. Bodic, G.L., J. Dunlop and J. Irvine, 2000. Virtual bus architecture for hierarchical cellular systems. Proceeding of the 11th IEEE International Symposium on Personal, Indoor and Mobile Radio Communications, Sept. 18-21, London, UK., pp: 861-865. DOI: 10.1109/PIMRC.2000.881545

5. Chen, J., 2001. SONET transcoder design for ATM over SONET or directly over fiber circuits and systems. Proceeding of International Symposium on ISCAS, May 6-9, Sydney, pp: 229-232. DOI: 10.1109/ISCAS.2001.921049

6. Hay, R., Comparing POS and ATM interfaces. Computer, 33: 102-103.

http://www2.computer.org/portal/web/csdl/doi/10.1 $109 / 2.863987$

7. Bernstein, G., B. Rajagopalan and D. Saha, 2003. Optical Network Control: Architecture, Protocols and Standards. Addison Wesley ProfessionalPearson Education, ISBN: 10: 0201753014, pp: 464.

8. Bakker, J.D. and R. Prasad, 1999. A multiple access protocol implementation for a virtual cellular network. Proceeding of 49th Conference on Vehicular Technology, May 16-20, IEEE Xplore Press, Houston, TX, USA., pp: 1707-1711. DOI: 10.1109/VETEC.1999.780702

9. IIwao Sasase, Multiple Access Protocol, Keio University, Japan.

http://www.sasase.ics.keio.ac.jp/jugyo/2005/Multip le\%20Access\%20Protocol.pdf 
10. Twu, D.C. and K.C. Chen, 1998. Design and analysis of the multiple access protocols over the CATV/HFC networks. Proceeding of 98th International Conference on Communications, June 7-11, Atlanta Georgia, pp: 854-858. DOI: 10.1109/ICC.1998.685132

11. Steven, S.G. and T. Wilson, 2002. Transparent Generic Framing Procedure (GFP): A protocol for efficient transport of block-coded data through SONET/SDH networks. IEEE Commun. Magaz., 40: 88-95. DOI: 10.1109/35.1000218

12. Scholten, M., E.Z. Zhenyu, H. Valencia and J. Hawkins, 2002. Data transport applications using GFP. IEEE Commun. Magaz., 40: 96-103. DOI: 10.1109/35.1000219

13. Valencia, E. H., M. Scholten and Z. Zhu, 2002. The Generic Framing Procedure (GFP): An overview. IEEE Commun. Magaz., 40: 63-71. DOI: $10.1109 / 35.1000215$
14. Eltahir, M. and J. Dunlop, 2001. Mapping 2.5/3G standards onto a virtual bus architecture for hierarchical cellular system. Proceeding of the 4th Conference on European Personal and Mobile Communications, Feb. 20-22, Vienna, Austria.

15. Varga, A., 2005. OMNeT++ discrete event simulation system, $\quad$ Version http://www.omnetpp.org/index.php?page=7

16. WTSA 2008. ITU Telecommunication Standardization Sector (ITU-T). http://www.itu.int/ITU-T 